\title{
MERIT: A MONOTONICALLY ERROR-BOUND IMPROVING TECHNIQUE FOR UNIMODULAR QUADRATIC PROGRAMMING
}

\author{
Mojtaba Soltanalian* and Petre Stoica \\ Dept. of Information Technology, Uppsala University, Uppsala, Sweden
}

\begin{abstract}
The NP-hard problem of optimizing a quadratic form over the unimodular vector set arises in radar code design scenarios as well as other active sensing and communication applications. To tackle this problem, a monotonically errorbound improving technique (MERIT) is proposed to obtain the global optimum or a local optimum of UQP with good sub-optimality guarantees. The provided sub-optimality guarantees are case-dependent and may outperform the $\pi / 4$ approximation guarantee of semi-definite relaxation.
\end{abstract}

Index Terms - Code design, radar codes, unimodular codes, quadratic programming, peak-to-average-power ratio (PAR)

\section{INTRODUCTION}

Unimodular codes are used in many active sensing and communication systems mainly as a result of the their optimal (i.e. unity) peak-to-average-power ratio (PAR). In a variety of applications, such as receiver signal-to-noise ratio (SNR) optimization, synthesizing cross ambiguity functions, steering vector estimation, and maximum likelihood (ML) detection, the code approximation can be formulated as the optimization of a quadratic form [1]-[8]. Therefore, we will study the problem

$$
\text { UQP: } \max _{\boldsymbol{s} \in \Omega^{n}} \boldsymbol{s}^{H} \boldsymbol{R} \boldsymbol{s}
$$

where $\boldsymbol{R} \in \mathbb{C}^{n \times n}$ is a given Hermitian matrix, $\Omega$ represents the unit circle, i.e. $\Omega=\{s \in \mathbb{C}:|s|=1\}$ and UQP stands for Unimodular Quadratic Program(ming).

In [9], the NP-hardness of UQP is proven by employing a reduction from an NP-complete matrix partitioning problem. Studies on polynomial-time (or efficient) algorithms for UQP have been extensive (e.g. see [9]-[22] and the references therein). In particular, the semi-definite relaxation (SDR) technique has been one of the most appealing approaches to the researchers. We refer the interested reader to the survey of the rich literature on SDR in [14].

\footnotetext{
This work was supported in part by the European Research Council (ERC) under Grant \#228044, and the Swedish Research Council.

* Please address all the correspondence to Mojtaba Soltanalian, Phone: (+46) 18-471-3168; Fax: (+46) 18-511925; Email: mojtaba.soltanalian@it.uu.se
}

Throughout the paper, we assume that $\boldsymbol{R}$ is positive semidefinite (if $\boldsymbol{R}$ is not positive semi-definite, one can make it so using a diagonal loading without changing the solution to (1)). We present the existing (analytically derived) sub-optimality guarantee for SDR. Let $v_{S D R}$ be the expected value of the UQP objective at the obtained randomized solution. Let $v_{o p t}$ represent the optimal value of the UQP objective. We have

$$
\gamma v_{o p t} \leq v_{S D R} \leq v_{o p t}
$$

with the sub-optimality guarantee coefficient $\gamma=\pi / 4$ [9][15]. For the sake of brevity, in the sequel the abbreviation SDR will be used for semidefinite relaxations followed by the randomization procedure.

Besides SDR, the literature does not offer many other numerical approaches to tackle UQP. In this paper, we propose a monotonically error-bound improving technique (called MERIT) that obtains the global optimum or a local optimum of UQP with generally good sub-optimality guarantees. MERIT provides real-time case-dependent sub-optimality guarantees $(\gamma)$ during its iterations. To the best of our knowledge, such guarantees for UQP were not known prior to this work. Using MERIT one may obtain better performance guarantees compared to the analytical worst-case guarantees (such as $\gamma=\pi / 4$ for SDR). The provided case-dependent sub-optimality guarantees are of practical importance in decision making scenarios.

\section{MERIT}

To help the formulation of MERIT, Theorem 1 presents a bijection among the set of matrices leading to the same solution.

Theorem 1. Let $\mathcal{K}(s)$ represent the set of matrices $\boldsymbol{R}$ for which a given $s \in \Omega^{n}$ is the global optimizer of UQP. Then

1. $\mathcal{K}(s)$ is a convex cone.

2. For any two vectors $s_{1}, s_{2} \in \Omega^{n}$, the one-to-one mapping

$$
\boldsymbol{R} \in \mathcal{K}\left(s_{1}\right) \Longleftrightarrow \boldsymbol{R} \odot\left(s_{0} \boldsymbol{s}_{0}^{H}\right) \in \mathcal{K}\left(s_{2}\right)
$$

(where $s_{0}=s_{1}^{*} \odot s_{2}$ ) holds among the matrices in $\mathcal{K}\left(s_{1}\right)$ and $\mathcal{K}\left(s_{2}\right)$. 


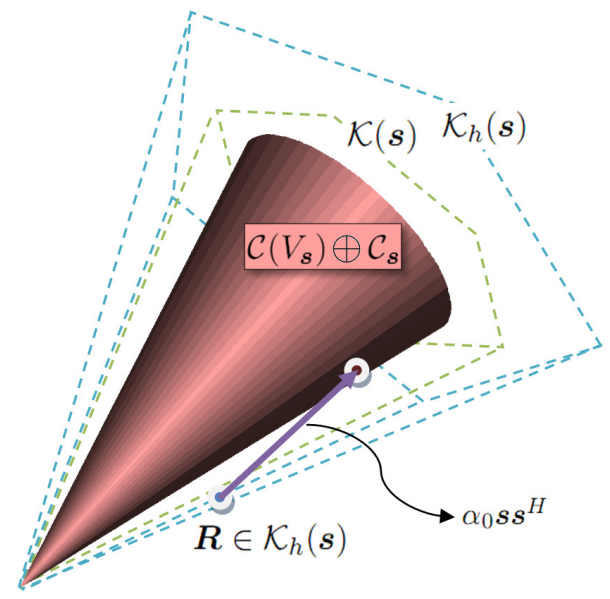

Fig. 1. An illustration of the result in Theorem 2. $\mathcal{K}_{h}(s)$ denotes the convex cone of matrices with $s$ as a stable point ${ }^{1}$ of the associated UQPs.

For proofs of the theorems in this paper, we refer the reader to [1]. It is interesting to note that in light of the above result, the characterization of the cone $\mathcal{K}(s)$ for any given $s=\widetilde{s}$ leads to a complete characterization of all $\mathcal{K}(s), s \in$ $\Omega^{n}$, and thus solving any UQP. While a complete tractable characterization of $\mathcal{K}(s)$ cannot be expected (due to the NPhardness of UQP), approximate characterizations of $\mathcal{K}(s)$ are possible. In the following, our goal is to provide an approximate characterization of the cone $\mathcal{K}(s)$ which can be used to tackle the UQP problem.

Theorem 2. For any given $s=\left(e^{j \phi_{1}}, \cdots, e^{j \phi_{n}}\right)^{T} \in \Omega^{n}$, let $\mathcal{C}\left(\boldsymbol{V}_{s}\right)$ represent the convex cone of matrices $\boldsymbol{V}_{s}=\boldsymbol{D} \odot$ $\left(s s^{H}\right)$ where $\boldsymbol{D}$ is any real-valued symmetric matrix with non-negative off-diagonal entries. Also let $\mathcal{C}_{s}$ represent the convex cone of matrices with s being their dominant eigenvector (i.e the eigenvector corresponding to the maximal eigenvalue). Then for any $\boldsymbol{R} \in \mathcal{K}(\boldsymbol{s})$, there exists $\alpha_{0} \geq 0$ such that for all $\alpha \geq \alpha_{0}$,

$$
\boldsymbol{R}+\alpha \boldsymbol{s} s^{H} \in \mathcal{C}\left(\boldsymbol{V}_{\boldsymbol{s}}\right) \oplus \mathcal{C}_{\boldsymbol{s}}
$$

where $\oplus$ stands for the Minkowski sum of the two sets.

An intuitive illustration of the result in Theorem 2 is shown in Fig. 1. Indeed, it can be shown that Theorem 2 is valid even when $s$ is a stable point ${ }^{1}$ of the UQP associated with $\boldsymbol{R}$. Finally, one can use $\mathcal{C}\left(\boldsymbol{V}_{\boldsymbol{s}}\right) \oplus \mathcal{C}_{\boldsymbol{s}}$ as an approximate characterization of $\mathcal{K}(s)$ noting that the accuracy of such a characterization can be measured by the minimal value of $\alpha_{0}$. Hereafter, we study a computational method to obtain an $\alpha_{0}$ which is as small as possible. We also formulate the

\footnotetext{
${ }^{1}$ The stationary points $(\boldsymbol{s})$ of an UQP associated with $\boldsymbol{R}$ can be characterized via the equation $\boldsymbol{R} \boldsymbol{s}=\boldsymbol{v} \odot \boldsymbol{s}$ where $\boldsymbol{v} \in \mathbb{R}^{n}$. If the vector $\boldsymbol{v}$ for a given stationary point $s$ was non-negative, then $s$ is a stable point of UQP. Any local optimum of UQP is also a stable point of UQP, see [1].
}

sub-optimality guarantee for a solution of UQP based on the above $\mathcal{K}(s)$ approximation

Using the previous results, we build a sequence of matrices (for which the UQP global optima are known) whose distance from the given matrix $R$ is decreasing. The proposed iterative approach can be used to solve for the global optimum of UQP or at least to obtain a local optimum (with an upper bound on the sub-optimality of the solution). We know from Theorem 2 that if $s$ is a stable point of the UQP associated with $\boldsymbol{R}$ then there exist matrices $\boldsymbol{Q}_{\boldsymbol{s}} \in \mathcal{C}_{\boldsymbol{s}}, \boldsymbol{P}_{\boldsymbol{s}} \in \mathcal{C}\left(\boldsymbol{V}_{\boldsymbol{s}}\right)$ and a scalar $\alpha_{0} \geq 0$ such that $\boldsymbol{R}+\alpha_{0} \boldsymbol{s s}^{H}=\boldsymbol{Q}_{\boldsymbol{s}}+\boldsymbol{P}_{\boldsymbol{s}}$. The latter equation can be rewritten as

$$
\boldsymbol{R}+\alpha_{0} \boldsymbol{s s ^ { H }}=\left(\boldsymbol{Q}_{\mathbf{1}}+\boldsymbol{P}_{\mathbf{1}}\right) \odot\left(s \boldsymbol{s}^{H}\right)
$$

where $Q_{1} \in \mathcal{C}_{1}, \boldsymbol{P}_{1} \in \mathcal{C}\left(V_{1}\right)$. We first consider the case of $\alpha_{0}=0$ which corresponds to the global optimality of $\boldsymbol{s}$. Consider the optimization problem:

$$
\min _{\boldsymbol{s} \in \Omega^{n}, \boldsymbol{Q}_{\mathbf{1}} \in \mathcal{C}_{\mathbf{1}}, \boldsymbol{P}_{\mathbf{1}} \in \mathcal{C}\left(\boldsymbol{V}_{\mathbf{1}}\right)}\left\|\boldsymbol{R}-\left(\boldsymbol{Q}_{\mathbf{1}}+\boldsymbol{P}_{\mathbf{1}}\right) \odot\left(\boldsymbol{s s}^{H}\right)\right\|_{F}
$$

Note that, as $\mathcal{C}_{\mathbf{1}} \oplus \mathcal{C}\left(\boldsymbol{V}_{\mathbf{1}}\right)$ is a convex cone, the global optimizers $\boldsymbol{Q}_{\mathbf{1}}$ and $\boldsymbol{P}_{\mathbf{1}}$ of (6) for any given $s$ can be easily found. On the other hand, the problem of finding an optimal $s$ for fixed $R_{1}=Q_{1}+P_{1}$ is non-convex and hence more difficult to solve globally (see below for details).

In the following, we introduce a suitable diagonal loading of $\boldsymbol{R}$ that is necessary to tackle (6). Next the optimization of the function in (6) is discussed through a separate optimization over the three variables of the problem. The detailed derivations can be found in [1].

- Diagonal loading of $\boldsymbol{R}$ : Let $\overline{\boldsymbol{R}}=\boldsymbol{R} \odot\left(\boldsymbol{s s}^{H}\right)^{*}$. We can compute $Q_{1}$ and $P_{1}$ (hence $R_{1}=Q_{1}+P_{1}$ ) for any initialization of $s$. In order to guarantee the positive definiteness of $\boldsymbol{R}_{\mathbf{1}}$, define $\varepsilon_{0} \triangleq\left\|\overline{\boldsymbol{R}}-\boldsymbol{R}_{\mathbf{1}}\right\|_{F}$. Then we diagonally load $\boldsymbol{R}$ with $\lambda>\lambda_{0}=-\sigma_{n}(\boldsymbol{R})+\varepsilon_{0}$ :

$$
\boldsymbol{R} \leftarrow \boldsymbol{R}+\lambda \boldsymbol{I} .
$$

- Optimization with respect to $Q_{1}$ : Let $\boldsymbol{R}_{Q}=\boldsymbol{R} \odot\left(s s^{H}\right)^{*}$ $\boldsymbol{P}_{\mathbf{1}}, H=\mathbf{1}_{n \times 1}^{T} \boldsymbol{R}_{Q} \mathbf{1}_{n \times 1}$, and

$\boldsymbol{Q}_{\mathbf{1}}(\rho) \triangleq \rho \boldsymbol{I}_{n}+\left(\boldsymbol{I}_{n}-\frac{\mathbf{1}_{n \times n}}{n}\right)\left(\boldsymbol{R}_{Q}-\rho \boldsymbol{I}_{n}\right)\left(\boldsymbol{I}_{n}-\frac{\mathbf{1}_{n \times n}}{n}\right)$.

Also let $\rho_{0}$ denote the maximal eigenvalue of $\boldsymbol{Q}_{\mathbf{1}}(0)$ corresponding to an eigenvector other than $\mathbf{1}_{n \times 1} / \sqrt{n}$. Then for fixed $P_{1}$ and $s$, the optimal solution $Q_{1}$ to (6) is given by

$$
Q_{1}=Q_{1}\left(\rho_{\star}\right)
$$

where

$$
\rho_{\star}=\left\{\begin{array}{cc}
\frac{H}{n} & \frac{H}{n} \geq \rho_{0}, \\
\rho_{0} & \text { otherwise. }
\end{array}\right.
$$


- Optimization with respect to $\boldsymbol{P}_{1}$ : For fixed $\boldsymbol{Q}_{1}$ and $s$, (6) can be rephrased as

$$
\min _{\boldsymbol{Q}_{\mathbf{1}} \in \mathcal{C}\left(\boldsymbol{V}_{\mathbf{1}}\right)}\left\|\boldsymbol{R}_{P}-\boldsymbol{P}_{\mathbf{1}}\right\|_{F}
$$

where $\boldsymbol{R}_{P}=\boldsymbol{R} \odot\left(s s^{H}\right)^{*}-\boldsymbol{Q}_{\mathbf{1}}$. The solution of (10) is simply given by

$$
\boldsymbol{P}_{\mathbf{1}}(k, l)= \begin{cases}\boldsymbol{R}_{P}^{\prime}(k, l) & \boldsymbol{R}_{P}^{\prime}(k, l) \geq 0 \text { or } k=l, \\ 0 & \text { otherwise, }\end{cases}
$$

where $\boldsymbol{R}_{P}^{\prime}=\Re\left\{\boldsymbol{R}_{P}\right\}$.

- Optimization with respect to $s$ : Define $\boldsymbol{R}_{\mathbf{1}}=\boldsymbol{Q}_{\mathbf{1}}+\boldsymbol{P}_{\mathbf{1}}$ and observe that $\left\|\boldsymbol{R}-\boldsymbol{R}_{\mathbf{1}} \odot\left(s s^{H}\right)\right\|_{F}^{2}$ can be rewritten as

$$
\begin{aligned}
& \left\|\boldsymbol{R}-\operatorname{Diag}(s) \boldsymbol{R}_{\mathbf{1}} \operatorname{Diag}\left(\boldsymbol{s}^{*}\right)\right\|_{F}^{2} \\
= & \operatorname{tr}\left(\boldsymbol{R}^{2}+\boldsymbol{R}_{\mathbf{1}}^{2}\right)-2 \Re\left\{\operatorname{tr}\left(\boldsymbol{R} \operatorname{Diag}(\boldsymbol{s}) \boldsymbol{R}_{\mathbf{1}} \operatorname{Diag}\left(\boldsymbol{s}^{*}\right)\right)\right\} .
\end{aligned}
$$

Moreover, it can be verified that [17]

$$
\operatorname{tr}\left(\boldsymbol{R} \operatorname{Diag}(s) \boldsymbol{R}_{\mathbf{1}} \operatorname{Diag}\left(s^{*}\right)\right)=s^{H}\left(\boldsymbol{R} \odot \boldsymbol{R}_{\mathbf{1}}^{T}\right) s .
$$

As $\boldsymbol{R} \odot \boldsymbol{R}_{1}^{T}$ is positive definite, we can employ the power method-like iterations introduced in the Appendix to decrease the criterion in (6), i.e. starting from the current $s=s^{(0)}$, a local optimum of the problem can be obtained by the iterations

$$
\boldsymbol{s}^{(t+1)}=e^{j \arg \left(\left(\boldsymbol{R} \odot \boldsymbol{R}_{1}^{T}\right) \boldsymbol{s}^{(t)}\right)} .
$$

Finally, the proposed algorithmic optimization of (6) based on the above results is summarized in Table 1-A. There exist examples for which the objective function in (6) does not converge to zero. As a result, the proposed method cannot obtain a global optimum of UQP in such cases. However, it is still possible to obtain a local optimum of UQP for some $\alpha_{0}>0$. To do so, we solve the optimization problem,

$$
\min _{\boldsymbol{s} \in \Omega, \boldsymbol{Q}_{\mathbf{1}} \in \mathcal{C}_{\mathbf{1}}, \boldsymbol{P}_{\mathbf{1}} \in \mathcal{C}\left(\boldsymbol{V}_{\mathbf{1}}\right)}\left\|\boldsymbol{R}^{\prime}-\left(\boldsymbol{Q}_{\mathbf{1}}+\boldsymbol{P}_{\mathbf{1}}\right) \odot\left(s s^{H}\right)\right\|_{F}
$$

with $\boldsymbol{R}^{\prime}=\boldsymbol{R}+\alpha_{0} \boldsymbol{s} \boldsymbol{s}^{H}$, for increasing $\alpha_{0}$. It is worth pointing out that achieving a zero value for the criterion in (15) implies $\boldsymbol{R}+\alpha_{0} \boldsymbol{s} \boldsymbol{s}^{H} \in \mathcal{K}(\boldsymbol{s})$. As a result, there exists a non-negative $\boldsymbol{v} \in \mathbb{R}^{n}$ such that $\left(\boldsymbol{R}+\alpha_{0} s s^{H}\right) \boldsymbol{s}=\boldsymbol{v} \odot \boldsymbol{s}$. Consequently, $\boldsymbol{R} \boldsymbol{s}=\left(\boldsymbol{v}-n \alpha_{0} \mathbf{1}\right) \odot s$ which implies $s$ is a stationary point of the UQP associated with $\boldsymbol{R}$.

The optimization problem in (15) can be tackled using the same tools as proposed for (6). In particular, note that increasing $\alpha_{0}$ decreases (15), see [1]. The obtained solution ( $\left.s, \boldsymbol{Q}_{\mathbf{1}}, \boldsymbol{P}_{\mathbf{1}}\right)$ of (6) can be used to initialize the corresponding variables in (15). In effect, the solution of (15) for any $\alpha_{0}$ can be used for the initialization of (15) with an increased $\alpha_{0}$.

Based on the above discussion and the fact that small values of $\alpha_{0}$ are of interest, a bisection approach can be used to obtain $\alpha_{0}$. The proposed method for obtaining a local optimum of UQP along with the corresponding $\alpha_{0}$ is described in Table 1-B. Using the proposed algorithm, the task of finding the minimal $\alpha_{0}$ can be accomplished within a finite number of steps [1].
Table 1. The MERIT Algorithm

(A) The case of $\alpha_{0}=0$

Step 0: Initialize the variables $Q_{1}$ and $P_{1}$ with $I$. Let $s$ be a random vector in $\Omega^{n}$.

Step 1: Perform the diagonal loading of $\boldsymbol{R}$ as in (7) (note that this diagonal loading is sufficient to keep $\boldsymbol{R}_{1}=\boldsymbol{Q}_{\mathbf{1}}+\boldsymbol{P}_{\mathbf{1}}$ always positive definite).

Step 2: Obtain the minimum of (6) with respect to $\boldsymbol{Q}_{\mathbf{1}}$ as in (8).

Step 3: Obtain the minimum of (6) with respect to $\boldsymbol{P}_{\mathbf{1}}$ using (11).

Step 4: Minimize (6) with respect to $s$ using (14).

Step 5: Goto step 2 until a stop criterion is satisfied, e.g. $\| \boldsymbol{R}-\left(\boldsymbol{Q}_{\mathbf{1}}+\right.$ $\left.\boldsymbol{P}_{\mathbf{1}}\right) \odot\left(\boldsymbol{s s}^{H}\right) \|_{F} \leq \epsilon_{0}$ (or if the number of iterations exceeded a predefined maximum number).

(B) The case of $\alpha_{0}>0$

Step 0: Initialize the variables $\left(\boldsymbol{s}, \boldsymbol{Q}_{\mathbf{1}}, \boldsymbol{P}_{\mathbf{1}}\right)$ using the results obtained by the optimization of (6) as in Table 1-A.

Step 1: Set $\delta$ (the step size for increasing $\alpha_{0}$ in each iteration). Let $\delta_{0}$ be the minimal $\delta$ to be considered and $\alpha_{0}=0$.

Step 2: Let $\alpha_{0}^{\text {pre }}=\alpha_{0}, \alpha_{0}^{\text {new }}=\alpha_{0}+\delta$ and $\boldsymbol{R}^{\prime}=\boldsymbol{R}+\alpha_{0}^{\text {new }} \boldsymbol{s s}^{H}$.

Step 3: Solve (15) using the steps 2-5 in Table 1-A.

Step 4: If $\left\|\boldsymbol{R}^{\prime}-\left(\boldsymbol{Q}_{\mathbf{1}}+\boldsymbol{P}_{\mathbf{1}}\right) \odot\left(\boldsymbol{s s}^{H}\right)\right\|_{F} \leq \epsilon_{0}$ do:

- Step 4-1: If $\delta \geq \delta_{0}$, let $\delta \leftarrow \delta / 2$ and initialize (15) with the previously obtained variables $\left(\boldsymbol{s}, \boldsymbol{Q}_{\mathbf{1}}, \boldsymbol{P}_{\mathbf{1}}\right)$ for $\alpha_{0}=\alpha_{0}^{\text {pre }}$. Goto step 2.

- Step 4-2: If $\delta<\delta_{0}$, stop.

Else, let $\alpha_{0}=\alpha_{0}^{\text {new }}$ and goto step 2 .

\subsection{Sub-optimality Analysis}

In this sub-section, we show how the proposed method can provide real-time sub-optimality guarantees and bounds during its iterations. Let $\alpha_{0}=0$ (as a result $\boldsymbol{R}^{\prime}=\boldsymbol{R}$ ) and define

$$
\boldsymbol{E} \triangleq \boldsymbol{R}^{\prime}-\underbrace{\left(\boldsymbol{Q}_{\mathbf{1}}+\boldsymbol{P}_{\mathbf{1}}\right) \odot\left(s s^{H}\right)}_{\boldsymbol{R}_{s}}
$$

where $\boldsymbol{Q}_{1} \in \mathcal{C}_{1}$ and $\boldsymbol{P}_{1} \in \mathcal{C}\left(V_{1}\right)$. By construction, the global optimum of the UQP associated with $\boldsymbol{R}_{\boldsymbol{s}}$ is $\boldsymbol{s}$. We have that

$$
\begin{aligned}
\max _{\boldsymbol{s}^{\prime} \in \Omega^{n}} \boldsymbol{s}^{\boldsymbol{H}^{H}} \boldsymbol{R} \boldsymbol{s}^{\prime} & \leq \max _{\boldsymbol{s}^{\prime} \in \Omega^{n}} \boldsymbol{s}^{{ }^{H}} \boldsymbol{R}_{\boldsymbol{s}} \boldsymbol{s}^{\prime}+\max _{\boldsymbol{s}^{\prime} \in \Omega^{n}} \boldsymbol{s}^{\prime H} \boldsymbol{E} \boldsymbol{s}^{\prime} \\
& \leq \max _{\boldsymbol{s}^{\prime} \in \Omega^{n}} \boldsymbol{s}^{\prime H} \boldsymbol{R}_{\boldsymbol{s}} \boldsymbol{s}^{\prime}+n \sigma_{1}(\boldsymbol{E}) \\
& =\boldsymbol{s}^{H} \boldsymbol{R}_{\boldsymbol{s}} \boldsymbol{s}+n \sigma_{1}(\boldsymbol{E})
\end{aligned}
$$

Furthermore,

$$
\begin{aligned}
\max _{\boldsymbol{s}^{\prime} \in \Omega^{n}} \boldsymbol{s}^{{ }^{H}} \boldsymbol{R} \boldsymbol{s}^{\prime} & \geq \max _{\boldsymbol{s}^{\prime} \in \Omega^{n}} \boldsymbol{s}^{\prime H} \boldsymbol{R}_{\boldsymbol{s}} \boldsymbol{s}^{\prime}+\min _{\boldsymbol{s}^{\prime} \in \Omega^{n}} \boldsymbol{s}^{\prime H} \boldsymbol{E} \boldsymbol{s}^{\prime} \\
& \geq \max _{\boldsymbol{s}^{\prime} \in \Omega^{n}} \boldsymbol{s}^{\prime H} \boldsymbol{R}_{\boldsymbol{s}} \boldsymbol{s}^{\prime}+n \sigma_{n}(\boldsymbol{E}) \\
& =\boldsymbol{s}^{H} \boldsymbol{R}_{\boldsymbol{s}} \boldsymbol{s}+n \sigma_{n}(\boldsymbol{E})
\end{aligned}
$$

As a result, an upper bound and a lower bound on the objective function for the global optimum of (6) can be obtained at each iteration. Next, suppose that we have to increase 

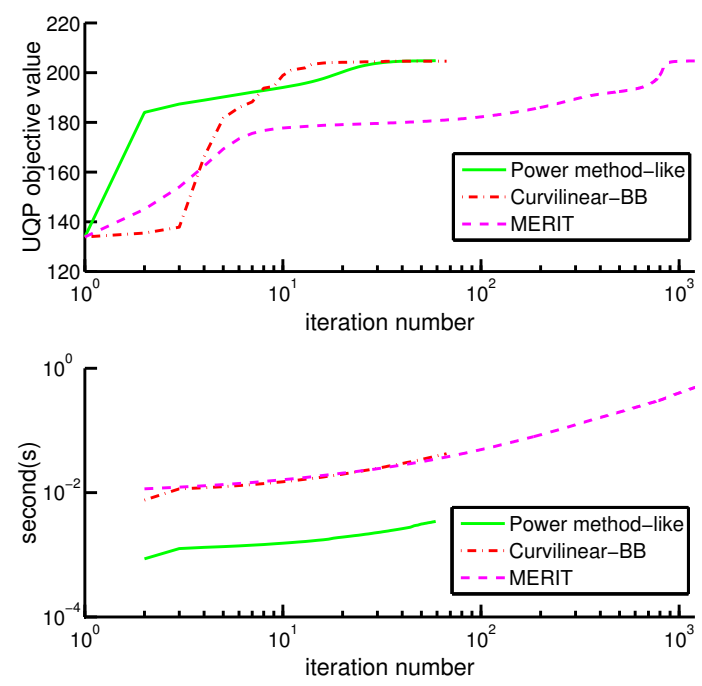

Fig. 2. A comparison of power method-like iterations, the curvilinear search of [18] with Barzilai-Borwein (BB) step size, and MERIT: (top) the UQP objective; (bottom) the required time for solving an UQP $(n=10)$ with same initialization.

$\alpha_{0}$ in order to obtain the convergence of $\|\boldsymbol{E}\|_{F}$ to zero. In such a case, we have that $\boldsymbol{R}=\boldsymbol{R}_{\boldsymbol{s}}-\alpha_{0} s \boldsymbol{s}^{H}$ and as a result, $\max _{\boldsymbol{s}^{\prime} \in \Omega^{n}} \boldsymbol{s}^{\prime H} \boldsymbol{R}_{\boldsymbol{s}} \boldsymbol{s}^{\prime}-\alpha_{0} n^{2} \leq \max _{\boldsymbol{s}^{\prime} \in \Omega^{n}} \boldsymbol{s}^{\prime H} \boldsymbol{R} \boldsymbol{s}^{\prime} \leq$ $\max _{\boldsymbol{s}^{\prime} \in \Omega^{n}} \boldsymbol{s}^{\prime H} \boldsymbol{R}_{\boldsymbol{s}} \boldsymbol{s}^{\prime}$, or equivalently,

$$
\boldsymbol{s}^{H} \boldsymbol{R}_{\boldsymbol{s}} \boldsymbol{s}-\alpha_{0} n^{2} \leq \max _{\boldsymbol{s}^{\prime} \in \Omega^{n}} \boldsymbol{s}^{\prime H} \boldsymbol{R} \boldsymbol{s}^{\prime} \leq \boldsymbol{s}^{H} \boldsymbol{R}_{\boldsymbol{s}} \boldsymbol{s} .
$$

The provided case-dependent sub-optimality guarantee is thus given by

$$
\gamma=\frac{\boldsymbol{s}^{H} \boldsymbol{R} \boldsymbol{s}}{\boldsymbol{s}^{H} \boldsymbol{R}_{\boldsymbol{s}} \boldsymbol{s}}=1-\frac{\alpha_{0} n^{2}}{\boldsymbol{s}^{H} \boldsymbol{R}_{\boldsymbol{s}} \boldsymbol{s}}=\frac{\boldsymbol{s}^{H} \boldsymbol{R} \boldsymbol{s}}{\boldsymbol{s}^{H} \boldsymbol{R} \boldsymbol{s}+\alpha_{0} n^{2}} .
$$

\section{NUMERICAL EXAMPLES AND DISCUSSION}

In order to examine the performance of the proposed method, several numerical examples will be presented. In all cases, we stopped the iterations when $\|\boldsymbol{E}\|_{F} \leq 10^{-9}$. Moreover, $\boldsymbol{R}$ is a random positive (semi)definite matrix; see [1] for details. We use the power method-like iterations discussed in Appendix A, and MERIT, as well as the curvilinear search of [18] with Barzilai-Borwein (BB) step size, to solve an UQP (with $n=10$ ) based on the same initialization. The resultant UQP objectives along with required times (in sec) versus iteration number are plotted in Fig. 2. It can be observed that the power method-like iterations approximate the UQP solution much faster than the curvilinear search of [18]. On the other hand, both methods are much faster than MERIT. This type of behavior, which is not unexpected, is due to the fact

\begin{tabular}{|c|l|l|l|l|}
\hline$n$ & $\begin{array}{l}\text { Rank } \\
(d)\end{array}$ & $\begin{array}{l}\text { \#problems for } \\
\text { which } \gamma=1\end{array}$ & Average $\gamma$ & $\begin{array}{l}\text { Average SDR time } \\
\text { Average MERIT time }\end{array}$ \\
\hline \hline $\mathbf{8}$ & 2 & 17 & 0.9841 & 1.08 \\
\cline { 2 - 5 } & 8 & 16 & 0.9912 & 0.81 \\
\hline \hline \multirow{2}{*}{$\mathbf{1 6}$} & 2 & 15 & 0.9789 & 2.08 \\
\cline { 2 - 5 } & 4 & 13 & 0.9773 & 0.95 \\
\cline { 2 - 5 } & 16 & 4 & 0.9610 & 0.92 \\
\hline
\end{tabular}

Table 2. Comparison of the performance of MERIT (see Table 1) and SDR [22] when solving the UQP for 20 random positive definite matrices of different sizes $n$ and ranks $d$.

that MERIT is not designed solely for local optimization; indeed, MERIT relies on a considerable over-parametrization in its formulation which is the cost paid for easily derivable sub-optimality guarantees. In general, one may employ the power method-like iterations to obtain a fast approximation of the UQP solution (e.g. by using several initializations), whereas for obtaining sub-optimality guarantees one can resort to MERIT.

Next, we approximate the UQP solutions for 20 full-rank random positive definite matrices of sizes $n \in\{8,16\}$. Inspired by [20] and [21], we also consider rank-deficient matrices $\boldsymbol{R}$ with rank $=d \ll n$. The performance of MERIT for different values of $d$ is shown in Table 2. Note that, in general, the provided sub-optimality guarantees $\gamma$ are considerably larger than $\pi / 4$ of SDR. We also employ SDR [22] to solve the same UQPs. In this example, we continue the randomization procedure of SDR until reaching the same UQP objective as for MERIT. A comparison of the computation times of SDR and MERIT can also be found in Table 2.

\section{A. APPENDIX: POWER METHOD FOR UQP}

Let $\left\{\boldsymbol{s}^{(t+1)}\right\}_{t=0}^{\infty}$ be a sequence of unimodular codes where $\boldsymbol{s}^{(t+1)}$ is the minimizer of the criterion $\left\|\boldsymbol{s}^{(t+1)}-\boldsymbol{R} \boldsymbol{s}^{(t)}\right\|_{2}$ for $\boldsymbol{s}^{(t+1)} \in \Omega^{n}$. The minimizing vector $\boldsymbol{s}^{(t+1)}$ of the latter criterion is simply given by the following power method-like iteration:

$$
\boldsymbol{s}^{(t+1)}=e^{j \arg \left(\boldsymbol{R s}^{(t)}\right)}
$$

Note that $\left\|\boldsymbol{s}^{(t+1)}-\boldsymbol{R} \boldsymbol{s}^{(t)}\right\|_{2}^{2}=$ const-2 $\Re\left\{\boldsymbol{s}^{(t+1) H} \boldsymbol{R} \boldsymbol{s}^{(t)}\right\}$. As a result, $\boldsymbol{s}^{(t+1)}$ is also the maximizer of the criterion $\Re\left\{\boldsymbol{s}^{(t+1) H} \boldsymbol{R} \boldsymbol{s}^{(t)}\right\}$. Moreover, if $\boldsymbol{s}^{(t+1)} \neq \boldsymbol{s}^{(t)}$ we have that

$$
\begin{aligned}
& \boldsymbol{s}^{(t+1) H} \boldsymbol{R} \boldsymbol{s}^{(t+1)}-\boldsymbol{s}^{(t) H} \boldsymbol{R} \boldsymbol{s}^{(t)} \\
= & \left(\boldsymbol{s}^{(t+1)}-\boldsymbol{s}^{(t)}\right)^{H} \boldsymbol{R}\left(\boldsymbol{s}^{(t+1)}-\boldsymbol{s}^{(t)}\right) \\
& +2 \Re\left\{\boldsymbol{s}^{(t+1) H} \boldsymbol{R} \boldsymbol{s}^{(t)}\right\}-2 \boldsymbol{s}^{(t) H} \boldsymbol{R} \boldsymbol{s}^{(t)}>0
\end{aligned}
$$

as $\Re\left\{\boldsymbol{s}^{(t+1) H} \boldsymbol{R} \boldsymbol{s}^{(t)}\right\}>\boldsymbol{s}^{(t) H} \boldsymbol{R} \boldsymbol{s}^{(t)}$. Therefore, the UQP objective is increasing through the power method-like iterations in (21). It can also be shown that the sequence $\left\{\boldsymbol{s}^{(t+1)}\right\}_{t=0}^{\infty}$ obtained by (21) converges to a local optimum (or a saddle point) of the UQP, see [1]. 


\section{B. REFERENCES}

[1] M. Soltanalian and P. Stoica, "Designing unimodular codes via quadratic optimization," IEEE Transactions on Signal Processing, Submitted, 2013.

[2] A. De Maio, S. De Nicola, Y. Huang, S. Zhang, and A. Farina, "Code design to optimize radar detection performance under accuracy and similarity constraints," IEEE Transactions on Signal Processing, vol. 56, no. 11, pp. $5618-5629$, Nov. 2008.

[3] A. De Maio and A. Farina, "Code selection for radar performance optimization," in Waveform Diversity and Design Conference, Pisa, Italy, June 2007, pp. 219-223.

[4] H. He, J. Li, and P. Stoica, Waveform Design for Active Sensing Systems: A Computational Approach. Cambridge, UK: Cambridge University Press, 2012.

[5] N. Levanon and E. Mozeson, Radar Signals. New York: Wiley, 2004.

[6] H. He, P. Stoica, and J. Li, "On synthesizing cross ambiguity functions," in IEEE International Conference on Acoustics, Speech and Signal Processing (ICASSP), Prague, Czech Republic, May 2011, pp. 3536-3539.

[7] J. Li and P. Stoica, Eds., Robust Adaptive Beamforming. NJ, USA: John Wiley \& Sons, Inc., 2005.

[8] J. Jaldén, C. Martin, and B. Ottersten, "Semidefinite programming for detection in linear systems - optimality conditions and space-time decoding," in IEEE International Conference on Acoustics, Speech, and Signal Processing (ICASSP), vol. 4, Hong Kong, April 2003, pp. 9-12.

[9] S. Zhang and Y. Huang, "Complex quadratic optimization and semidefinite programming," SIAM Journal on Optimization, vol. 16, no. 3, pp. 871-890, 2006.

[10] Z. Wen, D. Goldfarb, S. Ma, and K. Scheinberg, "Row by row methods for semidefinite programming," Industrial Engineering, pp. 1-21, 2009.

[11] S. Verdú, "Computational complexity of optimum multiuser detection," Algorithmica, vol. 4, pp. 303-312, 1989.

[12] W.-K. Ma, B.-N. Vo, T. Davidson, and P.-C. Ching, "Blind ML detection of orthogonal space-time block codes: efficient high-performance implementations," IEEE Transactions on Signal Processing, vol. 54, no. 2, pp. 738-751, feb. 2006.
[13] T. Cui and C. Tellambura, "Joint channel estimation and data detection for OFDM systems via sphere decoding," in IEEE Global Telecommunications Conference (GLOBECOM), vol. 6, Texas, USA, Dec. 2004, pp. 3656-3660.

[14] Z.-Q. Luo, W. K. Ma, A.-C. So, Y. Ye, and S. Zhang, "Semidefinite relaxation of quadratic optimization problems," IEEE Signal Processing Magazine, vol. 27, no. 3, pp. 20 -34, May 2010.

[15] A. So, J. Zhang, and Y. Ye, "On approximating complex quadratic optimization problems via semidefinite programming relaxations," Mathematical Programming, vol. 110, pp. 93-110, 2007.

[16] M. Kisialiou and Z.-Q. Luo, "Performance analysis of quasi-maximum-likelihood detector based on semidefinite programming," in IEEE International Conference on Acoustics, Speech, and Signal Processing (ICASSP), vol. 3. Pennsylvania, USA: IEEE, 2005, pp. iii-433.

[17] R. Horn and C. Johnson, Matrix Analysis. Cambridge, UK: Cambridge University Press, 1990.

[18] Z. Wen and W. Yin, "A feasible method for optimization with orthogonality constraints," Mathematical Programming, pp. 1-38, 2013.

[19] M. Soltanalian, B. Tang, J. Li, and P. Stoica, "Joint design of the receive filter and transmit sequence for active sensing," IEEE Signal Processing Letters, vol. 20, no. 5, pp. 423-426, 2013.

[20] A. T. Kyrillidis and G. N. Karystinos, "Rank-deficient quadratic-form maximization over M-phase alphabet: Polynomial-complexity solvability and algorithmic developments," in IEEE International Conference on Acoustics, Speech and Signal Processing (ICASSP), May 2011, pp. 3856-3859.

[21] G. Karystinos and A. Liavas, "Efficient computation of the binary vector that maximizes a rank-deficient quadratic form," IEEE Transactions on Information Theory, vol. 56, no. 7, pp. 3581-3593, July 2010.

[22] A. De Maio, Y. Huang, M. Piezzo, S. Zhang, and A. Farina, "Design of optimized radar codes with a peak to average power ratio constraint," IEEE Transactions on Signal Processing, vol. 59, no. 6, pp. 2683-2697, June 2011. 